\title{
Chinesische Medizin als Bestandteil einer integrativen Onkologie
}

\author{
Stefan Kirchhoff
}

\section{Zusammenfassung}

Die Chinesische Medizin findet in China und zunehmend auch im Westen Anwendung als adjuvante Therapie in der Onkologie. Innerhalb eines eigenen, von der Biomedizin unterschiedlichen Denkmodells beschreibt die TCM Pathomechanismen für die Tumorentstehung und daraus resultierende Therapiestrategien. Aufgrund einer, auf der direkten Wahrnehmung des Arztes beruhenden, individuellen Diagnose kommen Verfahren wie Phytotherapie, Akupunktur, Qi Gong und Diätetik zum Einsatz. Therapeutisches Ziel ist nicht die direkte Bekämpfung des Tumors, sondern die Stärkung des Patienten und seines Immunsystems sowie die psychische Stabilisierung. Eingesetzt wird die TCM in der begleitenden Tumortherapie, als prä- und postoperative Behandlung und insbesondere in der Therapie von Nebenwirkungen von Chemotherapie und Radiatio.

\section{Geschichtliche Entwicklung}

Um die Möglichkeiten und Grenzen der Chinesischen Medizin im Verständnis und der Therapie von malignen Tumoren einschätzen zu können, ist zunächst eine Begriffsdefinition und Abgrenzung der Begriffe Chinesische Medizin und Traditionelle Chinesische Medizin (TCM) erforderlich. Während die Bezeichnung „Chinesische Medizin“ häufig benutzt wird, um sämtliche Theorien, Konzepte und Methoden der Medizin Chinas seit ihren zurückverfolgbaren Anfängen zusammenzufassen, steht „Traditionelle Chinesische Medizin (TCM)“ für ein Kunstprodukt der letzten 60 Jahre, das, nicht zuletzt aus politischem Kalkül, aus den dominierenden medizinischen Lehrmeinungen der Chinesischen Medizin geschaffen und ideologisch bereinigt wurde [9].

Die TCM als offizielle Variante der Chinesischen Medizin besitzt mittlerweile auch eigene onkologische Abteilungen an chinesischen Kliniken wie z.B. dem Sino-Ja-
Schlüsselwörter

Chinesische Medizin, TCM, Qi, Akupunktur, Phytotherapie

panese Friendship Hospital in Beijing. Seit 1949 wird unter anderem auch auf nationaler Ebene Forschung zum Thema Krebs und TCM betrieben. Des Weiteren wird die TCM auch permanent durch Einflüsse der westlichen Biomedizin weiterentwickelt und modifiziert. Einzelne Arzneidrogen der chinesischen Materia Medica werden inzwischen nicht mehr nur aufgrund ihrer klassischen Wirkbeschreibungen verordnet, sondern auch teilweise ausschließlich aufgrund westlich-pharmakologischer Erkenntnisse.

Die klassische chinesische Medizin, aus der die TCM hervorgegangen ist, hatte keinen eigenständigen Begriff für Krebs und somit auch kein karzinomspezifisches Konzept. Allerdings wurden schon früh Tumoren als Verhärtungen, Schwellungen und Masseansammlungen mit unterschiedlichen Begriffen beschrieben und Behandlungsstrategien entwickelt. In der Shang-Dynastie tauchte ca.1100 v.Chr. der Begriff Liu in Knocheninschriften auf. Er bedeutet soviel wie „beulenartige Geschwulst“. Fast 2000 Jahre später in der Song-Dynastie wurde erstmalig das
Schriftzeichen ai als Bezeichnung für eine bedrohliche tumoröse Erkrankung im Sinne von Krebs verwendet (Wei Ji Bao Shu 1171).

In dem Klassiker Ren Zhai Zhi Zhi Fu Yi Fang wird ai als „perforierend und einwärts penetrierend" bezeichnet, was auffällige Parallelen zum biomedizinischen Verständnis von einem infiltrativen und metastasierenden Wachstum zeigt (Li Peiwen [5]). Neben liu und ai gab es noch eine Vielzahl anderer Schriftzeichen, die je nach Beschaffenheit und Wachstum unterschiedliche Tumore beschrieben.

\section{Pathophysiologie}

Die medizintheoretischen Konzepte der Chinesischen Medizin zu Krankheitsentstehung, Diagnostik und Therapie unterscheiden sich zunächst deutlich von der Sichtweise der Biomedizin.

Die Chinesische Medizin ist in erster Linie eine Medizin der Informationsverarbeitung, der ein energetisches Lebenskraftkonzept Qi zugrunde liegt [3]. Auf den menschlichen Organismus bezogen bedeutet Gesundheit ein freies und gleichmäßiges Zirkulieren dieses Qi durch den Körper und die Akupunkturleitbahnen. Krankheit entsteht zum Beispiel, wenn $Q i$ in einzelnen Funktionskreisen in einen Mangel gerät und seine Funktion der Aufrechterhaltung der physiologischen Körperfunktionen nicht mehr erfüllen kann oder wenn $Q i$ in seinem Fluss stagniert und akkumuliert. Daraus ergeben sich zwei der wichtigsten Pathomechanismen der Karzinomentstehung aus Sicht der Chinesischen Medizin. Der Mangel von Qi kann teilweise mit der westlichen Vorstellung einer geschwächten Immunabwehr in Einklang gebracht werden, während die Stase oder Stagnation die Ansammlung von tumoröser Masse beschreibt. Eine 
weitere allgemeine Aussage zur Tumorentstehung ist, dass zu einem Mangel an physiologischem, gesundem Qi noch ein Überwiegen von „schädlichem“ Qi kommen muss. Als schädliches Qi kann man unter anderem den Einfluss von Toxinen, Viren und anderen kanzerogenen Substanzen betrachten.

Die Chinesische Medizin fasst die Krankheitsursachen in drei großen Gruppen zusammen. Es gibt äußere, innere und sonstige Krankheitsursachen [6]. Zu den äußeren Krankheitsursachen zählen ursprünglich klimatische Einflüsse wie Kälte, Feuchtigkeit und Wind. Unter dem Einfluss der Biomedizin werden mittlerweile teilweise auch andere, von außen in den Organismus eindringenden Faktoren wie Bakterien, Viren, Allergene und Toxine als äußere Krankheitsursachen betrachtet. Als innere Krankheitsursachen betrachtet die Chinesische Medizin emotionale Probleme, welche z.B. durch Unterdrückung oder Verdrängung zu Stagnationen führen können. Emotional bedingte Blockaden spielen aus Sicht der Chinesischen Medizin bei der Krebsentstehung eine nennenswerte Rolle [4]. $\mathrm{Zu}$ den sonstigen Krankheitsursachen zählt man u.a. Fehlernährung.

Die klinische Bedeutung einer differenzierten Betrachtung der Krankheitsursachen ergibt sich innerhalb der Chinesischen Medizin aus den daraus resultierenden Empfehlungen für eine entsprechende Lebensführung mit konkreten Maßnahmen wie Ernährungsverhalten, Bewegungs- und Meditationsübungen zur Prävention.

In der Chinesischen Medizin gibt es noch weitere Pathomechanismen, die in unterschiedlichen Kombinationen zur Tumorenstehung beitragen können. Neben dem Mangel und der Qi-Stagnation zählen noch „Blutstase“ und „Akkumulation von Schleim“ als zwei bedeutende Mechanismen dazu. Das Letztere ist als Konzept deshalb von Bedeutung, weil Qi, als durch die Akupunkturleitbahnen zirkulierendes Lebenskraftkonzept, nicht substanziell fassbar ist. Nach chinesischer Vorstellung akkumulieren als Folge länger anhaltender Stagnationen „trübe Substanzen“. Diese verdichten sich zu überschüssiger Materie und werden in der Chinesischen Medizin dann als „Schleim“ bezeichnet. „Schleim“ ist ein überaus differenziertes Konzept der Chinesischen Medizin und korreliert unter anderem auch mit benignen Tumoren und der Adipositas, welche teilweise ja auch als Risikofaktor für die Tumorentstehung eingestuft wird. Auf eine detaillierte Darstellung aller Pathomechanismen wird an dieser Stelle verzichtet, da im Rahmen einer integrativen Tumortherapie im Westen meist nicht die Behandlung des Tumors im Vordergrund steht, sondern vielmehr eine konstitutionelle Unterstützung oder Stärkung des Patienten sowie die Behandlung der Folgen der konventionellen onkologischen Therapie.

Alle erwähnten Krankheitsursachen und Pathomechanismen sind allerdings nicht tumorspezifisch, sondern gelten auch für eine Vielzahl anderer Erkrankungen wie z.B. benigne Tumoren, chronische degenerative Erkrankungen oder auch psychosomatische und psychiatrische Erkrankungen.

\section{Diagnostik}

Um mit Methoden der Chinesischen Medizin nach den Regeln der Chinesischen Medizin behandeln zu können, bedarf es einer vorhergehenden Diagnose innerhalb des anzuwendenden Medizinsystems. Auch hier gibt es deutliche Unterschiede zur abendländischen Biomedizin. In der Diagnostik zeigen sich sowohl Schwächen als auch Stärken der Chinesischen Medizin. Bei der Chinesischen Medizin handelt es sich, anders als bei der quantitativ

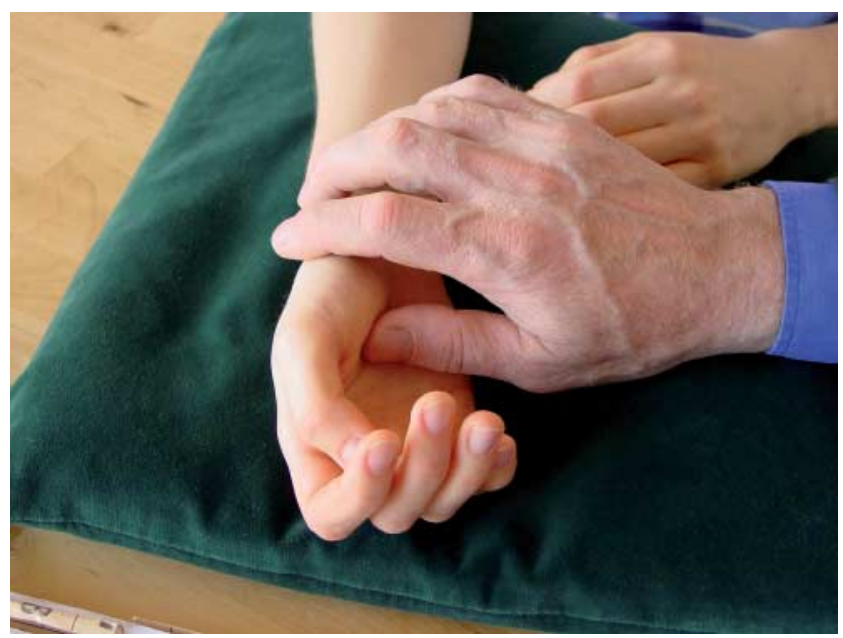

orientierten Biomedizin, primär um eine qualitative Medizin. Es wird nichts gemessen. Die subjektive Befindlichkeit des Patienten mit seinen Symptomen und die, zumindest bislang, nicht messbaren Pulsqualitäten (Abb.1) sowie das Aussehen der Zunge sind für die Diagnosefindung entscheidend. Man erfasst eher die Gesamtheit des Patienten, in die eingebunden sich der Tumor entwickelt hat, als die Natur des Tumors als solche.

Die Stärke der chinesischen Diagnostik liegt darin, dass die diagnostischen Verfahren der Befragung, Inspektion und Palpation über die Jahrtausende so differenziert wurden, dass bis zu 27 Pulsqualitäten an 12 unterschiedlichen Taststellen des Radialispulses unterschieden werden und jede Veränderung des Zungenkörpers in Form und Farbe sowie der Belag interpretiert werden. Dadurch erlangt man einen hohen Individualisierungsgrad mit zahlreichen Subgruppen für die einzelnen, im System der Biomedizin klar differenzierten Tumorarten. Bedeutsamer für eine integrative Tumortherapie ist aber die Möglichkeit der chinesischen Diagnostik, sowohl die Konstitution des Patienten zu bestimmen als auch die durch die Grunderkrankung oder auch durch die konventionelle Therapie geschwächten Aspekte des physiologischen Qi zu erkennen. Die chinesische Diagnoseformulierung stellt gleichzeitig auch immer eine Handlungsanweisung dar. Somit hat die weitere Differenzierung von Tumorpatienten in Subgruppen der TCM auch eindeutig definierte therapeutische Konsequenzen.
Abb. 1: Die Pulsuntersuchung ist ein wesentlicher Bestandteil der chineischen Diagnostik. Foto: Klinik am Steigerwald, Gerolzhofen. 
Der Nachteil der chinesischen Diagnostik ist, dass sie auf die Anamnese und die vom Behandler direkt wahrnehmbaren Tast- und Inspektionsbefunde limitiert ist. Die Chinesische Medizin kann somit zwar ein auftretendes Ungleichgewicht beim Patienten sehr früh erkennen, aber nicht ein Karzinom als solches diagnostizieren. Für die Diagnosestellung Krebs sind die diagnostischen Verfahren der Biomedizin unabdingbar.

\section{Therapie}

Ziel jeder Behandlung mit Chinesischer Medizin ist eine weitestgehende Wiederherstellung des energetischen Gleichgewichts. Im besonderen Fall der Onkologie, in dem ein Erreichen dieser gesunden Harmonie häufig nicht möglich ist, bedeutet das eine Verbesserung der Lebensqualität im Allgemeinen, körperliche Kräftigung, psychische Stabilisierung, Stimulierung des Immunsystems und darüber eine eventuelle Verkleinerung des Tumors, Schmerzreduktion, Reduktion der Nebenwirkungen der konventionellen Therapie und Erhöhung der Überlebensdauer. Auch die Prävention von Rezidiven ist ein wichtiger Ansatz. Voraussetzung für eine erfolgreiche Therapie und das Erreichen eines oder mehrerer dieser Therapieziele ist eine korrekte chinesische Diagnose, die den Zustand des Patienten individuell erfasst. Eine Standardtherapie z.B. zur Immunstärkung eines Patienten gibt es nicht.

Als therapeutische Verfahren kommen primär die chinesische Phytotherapie und die Akupunktur zum Einsatz; aber auch Qi Gong und die chinesische Ernährungstherapie werden angewendet. Die Phytotherapie kann im Vergleich zur Akupunktur besser Mangelzustände beheben und ausgeprägte Formen von Blutstase und Schleimakkumulation lösen. Die Akupunktur hingegen bewährt sich in der Schmerztherapie, der psychischen Stabilisierung und der Behandlung einiger Nebenwirkungen wie z.B. der Übelkeit bei Chemotherapie.

Das Qi Gong, als ein Verfahren mit Bewegungs- und Atemübungen sowie meditativen Elementen, wird besonders zur psychischen Stabilisierung und zur Stärkung der Immunfunktionen verordnet.

Yi Qi Huo Xue Xiao Pi Yin (Gui Yang TCM-Universität)

\begin{tabular}{|l|l|l|l|}
\hline Huangqi & Astragali radix & Tragantwurzel & $15 \mathrm{~g}$ \\
\hline $\begin{array}{l}\text { Baihuasheshecao } \\
\text { Chaihu }\end{array}$ & Hedyotidis herba & Hedyotiskraut & $15 \mathrm{~g}$ \\
\hline Xiangfu & Bupleuri radix & chinesische Hasenohrwurzel & $12 \mathrm{~g}$ \\
\hline $\begin{array}{l}\text { Danshen } \\
\text { Baishao }\end{array}$ & Cyperi rhizoma & Nussgraswurzelstock & $12 \mathrm{~g}$ \\
\hline Baijiezi & Paeoniae lactiflorae radix & wotwurzelsalbeiwurzel & $12 \mathrm{~g}$ \\
\hline Wangbuliuxing & Sinapis alba semen & weißer Senfsamen & $12 \mathrm{~g}$ \\
\hline Danggui & Vaccariae segetalis semen & Vaccaria-Samen & $12 \mathrm{~g}$ \\
\hline Haizao & Angelicae sinensis radix & chin. Engelwurz-Wurzel & $12 \mathrm{~g}$ \\
\hline
\end{tabular}

Die chinesische Diätetik erfüllt auf schwächere Art ähnliche Funktionen wie die Phytotherapie.

In der TCM gibt es zwei grundlegende Strategien zur Behandlung von Tumorpatienten. Die erste Strategie besteht primär in der Behandlung der Grunderkrankung selbst. Bei der zweiten Strategie stehen die Behandlung der unerwünschten Wirkungen der konventionellen Therapie und die zunehmende Schwäche des Patienten im Vordergrund.

Für welche dieser beiden Strategien man sich entscheidet oder ob man eine Kombination beider wählt, hängt von verschiedenen Faktoren ab. Dazu zählen u.a. das Stadium der Erkrankung, die Konstitution und der aktuelle Zustand des Patienten und das Auftreten sowie Art und Stärke von Nebenwirkungen von Chemotherapie oder Radiatio.

Für die Behandlung der Grunderkrankung gibt es wiederum vier Hauptstrategien, die auf die oben erwähnten Pathomechanismen zurückzuführen sind [5]:

1. Stärkung des physiologischen Qi

2. Blut beleben und Blutstase transformieren

3. Hitze klären und Toxine ausleiten

4. Schleim transformieren und Verhärtungen aufweichen

Folgende Rezeptur für die adjuvante Therapie einer Form des Mammakarzinoms stellt beispielhaft das Vorgehen in der chinesischen Arzneimitteltherapie vor. Hierbei ist zu beachten, dass es sich bei dieser Rezeptur nicht um eine allgemein- gültige Kombination für Mammakarzimone handelt, sondern, wie oben erwähnt, nur aufgrund einer chinesischen Diagnose für Patientinnen mit einem bestimmten Syndrommuster geeignet ist (s. Tabelle).

Die Wirkung der Rezeptur wird aus Sicht der TCM als „Qi tonisierend, Blut nährend, Qi bewegend sowie Blutstase und Verhärtungen lösend“ beschrieben. Ihr wird somit eine die Patientin und ihr Immunsystem stärkende Wirkung wie auch eine gewisse antitumoröse Wirkung zugeschrieben.

Astragali radix hat in der TCM eine das Qi tonisierende Wirkung. In Tierversuchen zeigte sich eine immunstimulierende, hepatoprotektive, antiinflammatorische sowie eine die nephrotoxische Wirkung von Chemotherapeutika reduzierende Wirkung [6]. Angelicae sinensis radix nährt und bewegt das Blut. Hier konnte eine die Phagozytose stimulierende Wirkung nachgewiesen werden. Den Qi-bewegenden Aspekt der Rezeptur stellen u.a. Bupleuri radix und Cyperi rhizoma dar. Vaccariae semen löst Blutstasen und reduziert Schwellungen speziell im Brustbereich. Auch Salviae miltiorhizae radix hat eine das Blut bewegende Eigenschaft. Sinapis alba semen und Sargassi thallus werden zur Transformation von Schleimakkumulation verordnet, wobei das erste gegen „kalten“ und das zweite gegen „heißen“ Schleim wirkt [1].

Das oben genannte Beispiel macht einige typische Charakteristika der chinesischen Arzneimitteltherapie im Allgemein und in der Onkologie deutlich.

Es werden fast ausschließlich Rezepturen, also Vielstoffgemische, verordnet 


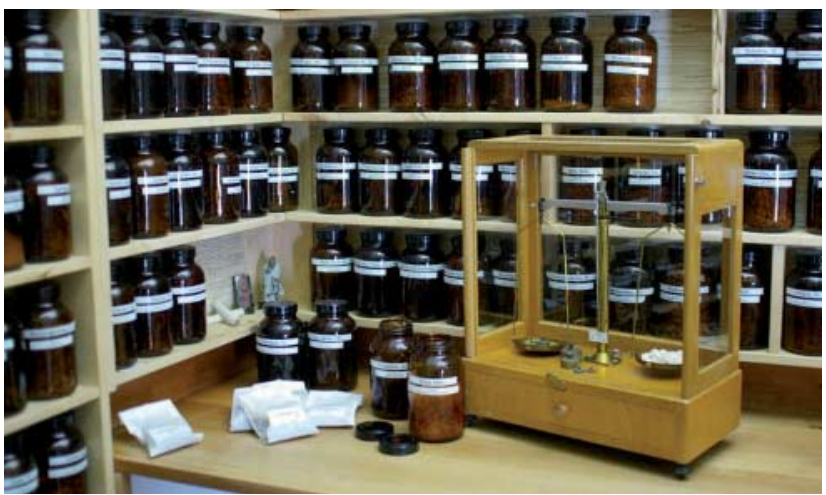

Abb. 2: In der Kräuterapotheke werden die Rezepturen zusammengestell, die in der chinesischen Phytotherapie hauptsächlich eingesetzt werden. Foto: Klinik am Steigerwald, Gerolzhofen.

(Abb.2). Einzelkräuter kommen kaum zum Einsatz. Dies resultiert aus dem ganzheitsorientierten Ansatz der TCM, in dem man chronische Erkrankungen meist auf das gleichzeitige Wirken mehrerer Pathomechanismen zurückführt. Diese müssen dann auch in der Therapiestrategie wieder zu finden sein. Außerdem gibt es bekannte und bewusst genutzte Interaktionen zwischen einzelnen Kräutern, die zu einer Wirkungsverstärkung führen können oder aber auch unerwünschte Reaktionen einer Droge durch eine andere neutralisieren. Durch die Verwendung mehrerer Kräuter mit ähnlichen Wirkungen können die Dosierungen der Einzelkräuter gering gehalten werden, um somit Überdosierungen und daraus resultierende unerwünschte Wirkungen zu verhindern.

Die Zusammenstellung einer chinesischen Rezeptur berücksichtigt die Wirkqualitäten der einzelnen Kräuter wie Geschmack, thermische Wirkung und Funktionskreisbezug. Auch bei Hinzufügen einzelner Kräuter aufgrund biomedizinischpharmakologischer Erkenntnisse wird berücksichtigt, ob die Substanz aufgrund ihrer Wirkqualitäten in das Gesamtbild passt.

So besitzen die stärkenden Arzneien meist einen süßen Geschmack und die ausleitenden Kräuter oftmals einen bitteren Geschmack. Je nach Rötung des Zungenkörpers und anderer Symptome in Bezug auf Hitze oder Kälte werden kühlende Kräuter wie z.B. Enzianwurzel oder wärmende Arzneien wie Senfsamen verordnet. Je nach Lokalisation der Symptomatik und Zugehörigkeit zu einzelnen Organfunktionen werden Drogen mit dem entsprechenden Funktionskreisbezug ge- wählt. So entsteht eine individualisierte Rezeptur für den Patienten. Die in der zeitgenössischen und klassischen Literatur erwähnten Rezepturen verstehen sich als Basisrezepturen, die je nach Befund in Dosis und Zusammensetzung modifiziert werden. Die aktuell in verschiedenen Zentren betriebene Wirkstoffsuche aus chinesischen Heilpflanzen deutet viel versprechende Ergebnisse hinsichtlich der Wachstumshemmung von Tumorzellen an [2]. Allerdings ist die Anwendung von Monosubstanzen ohne Berücksichtigung des übergeordneten Harmoniemusters nicht mehr als Therapie im Rahmen der TCM zu betrachten.

Ein operativer Eingriff stellt für den Patienten oft eine Belastung auf unterschiedlichen Ebenen dar. Auch in der präund postoperativen Begleitung bietet die chinesische Medizin therapeutische Konzepte an. Nach der Vorstellung der TCM sollte ein Patient vor einer Operation so gestärkt und psychisch stabil wie möglich sein. Ein intraoperativer Blutverlust kann Qi und Blut stark schwächen, die Regeneration nach dem Eingriff erfordert Qi und die OP als Trauma kann aus Sicht der TCM Stagnationen der Qi- und Blut-Zirkulation erzeugen.

Eine Beispielrezeptur für eine präoperative Stärkung von Qi und Blut ist Shengyutang (Zhu Dan Xi). Für die Stärkung bei einem dominierenden Yin-Mangel wird häufig Xiao Ying Tang (Jing Yue Quan Shu) verordnet. Zur präoperativen psychischen Stabilisierung wird die klassiche Rezeptur Gui Pi Tang empfohlen. Die postoperative Regeneration des Patienten kann mit Rezepturen wie Shi Quan Da Bu Wan oder anderen gefördert werden [4].
Eine der häufigsten Indikationen der TCM im Kontext der Onkologie ist im Westen die Behandlung der Nebenwirkungen von Chemotherapie und Radiatio. Auch hier gilt, dass eine Differenzierung nach den Regeln der TCM vor der Therapie erforderlich ist. Die durch die Chemotherapie verursachten Symptome müssen entsprechend interpretiert werden.

Die auftretenden Nebenwirkungen können grob in vier Gruppen unterteilt werden [7]:

1. Mangel an Qi, Blut und Yin (z.B. Haarausfall, Erschöpfung, Diarrhoe, Appetitlosigkeit, Parästhesien)

2. Rebellierendes Magen-Qi (z.B. Übelkeit, Erbrechen)

3. Magen-Hitze (Stomatitis, Ulcera)

4. Blut-Hitze (z. B. Hämaturie, Fieber, Zystitis)

Aufgrund dieser Klassifikation werden dann Kräuter mit Qi tonisierender (z. B. Astragalus radix), Magen-Qi absenkender Wirkung (z.B. Pinellia rhizoma), MagenHitze kühlende Arzneien (z.B. Phragmitis communis rhizoma) oder Blut-Hitze kühlende Kräuter (z.B. Moutan radicis cortex) verordnet.

Die Akupunktur hat sich ebenfalls als sinnvolle Begleittherapie erwiesen, insbesondere bei der Chemotherapie induzierten Übelkeit, bei der u.a. die Punkte Ren Mai 13, Magen 36, Magen 34 und der in Studien gut belegte Perikard 6 genadelt werden [8]. Aber auch für die Behandlung von Erschöpfung, Knochenmarkssuppression, Zystitis und Stomatitis gibt es konkrete Empfehlungen.

\section{Fazit}

Die Chinesische Medizin bietet als eigenständiges Medizinsystem mit einem der Biomedizin unterschiedlichen Diagnoseund Therapiesystem eine sinnvolle Ergänzung zur konventionellen Behandlung. Während die Chinesische Medizin nicht in der Lage ist, eine maligne Erkrankung sicher als solche zu diagnostizieren, liegt ihre Stärke in der individuellen Differenzierung aller beim Patienten auftretenden Symptome inklusive Veränderungen des 
Zungenbildes und der Pulsqualitäten. Diese führt zu einer Syndromdiagnose, welche die Konstitution des Patienten mit berücksichtigt. Die Ergänzung durch die TCM liegt darin, dass die therapeutischen Maßnahmen nicht primär auf die Vernichtung des Tumors ausgerichtet ist, sondern die Stärkung und Harmonisierung der physiologischen Funktionen, insbesondere des Immunsystems betont. Eine kompe- tente Anwendung der TCM ist aber nur innerhalb ihres Systems möglich. Dabei bedient sich die TCM einer eigenen Terminologie und eigener Theorien zu den Krankheitsmechanismen und Therapiestrategien. Eine Einbindung der TCM in die onkologische Therapie im Westen erfordert somit die Bereitschaft, sich mit diesen Theorien auseinanderzusetzen. Die Datenlage ist noch unbefriedigend. Auch wenn es in China zahlreiche positive Untersuchungsergebnisse gibt, ist deren Wert jedoch vielfach nur schwer zu beurteilen. Bei korrekter Anwendung handelt es sich bei der TCM um eine kostengünstige und besonders nebenwirkungsarme Therapieoption, die in der Anwendung und weiteren Erforschung in Hinblick auf die Onkologie Beachtung finden sollte.

\section{Literatur}

[1] Bensky D, Clavey S, Stöger E: Chinese Herbal Medicine. Materia Medica. 3rd Edition. Seattle: Eastland Press; 2004.

[2] Efferth T, Kahl S, Paulus K, Adams M, Rauh R, Boechzelt H, Hao X, Kaina B, Bauer R: Phytochemestry and Pharmacogenomics of natural product derived from traditional chinese medica with activity against tumor cells. Molecular Cancer Therapy. 2008; 7 (1): 152.

[3] Kubny M: Qi-Lebenskraftkonzepte in China. Heidelberg: Haug Verlag; 1995.

[4] Li J: Breast Cancer in TCM. Seminarmitschrift Universität Witten/Herdecke; 2008.

[5] Li P, Cheng Z, Du X: Management of Cancer with Chinese Medicine. St. Albans, UK: Donica Publishing Ltd; 2004.

[6] Maciocia G: Die Grundlagen der Chinesischen Medizin. Kötzting: VGM Verlag Dr. Erich Wühr; 1997.

[7] Maciocia G: Chemotherapy and Chemo-Support; 1999.

[8] Molassiotis A, Helin AM, Dabbour R, Hummerston S: The effects of P6 acupressure in the prophylaxis of chemotherapy-related nausea and vomiting in breast cancer patients. Complement Ther Med. 2007; 15(1): 3-12.

[9] Ots T: Medizin und Heilung in China. Berlin: Dietrich Reimer Verlag; 1987.

\section{Su m mary}

In China, and increasingly in the West as well, Chinese Medicine is being used as an adjuvant therapy in oncology. Within an independent system, completely different from biomedical sciences, Traditional Chinese Medicine describes pathomechanisms for the development of tumours and the therapy strategies deriving from the same. The application of treatment methods such as Chinese Herbal Medicine, Acupuncture, Qi Gong and Dietetics depends on the individual diagnosis based on the personal perception of the medical doctor. In fact, strengthening the patient and his immune system as well as the mental stabilisation are the therapeutical intentions rather than the direct fight against the tumour. Traditional Chinese Medicine is used as a collateral tumour therapy, as a preoperative or postoperative treatment, and particularly within the therapy of side effects of chemotherapy and radiotherapy.

Keywords

Chinese Medicine, TCM, Qi, acupuncture, herbal medicine 